\title{
Inhibitory Effects of Antioxidant Reagent in Reactive Oxygen Species Generation and Penetration of Appressoria of Alternaria alternata Japanese Pear Pathotype
}

\author{
Gang-Su Hyon, Ken-ichi Ikeda, Naoki Hosogi, Takeshi Shinogi, and Pyoyun Park
}

First, fourth, and fifth authors: Laboratory of Stress Cytology, Graduate School of Agricultural Science, and second author: Organization of Advanced Science and Technology, Kobe University, 1-1 Rokkodai, Nada, Kobe 657-8501, Japan; and third author: Laboratory of NanoStructure Physiology, Okazaki Institute for Integrative Bioscience, 5-1 Higashiyama, Myodaiji, Okazaki, 444-8787, Japan. Accepted for publication 5 April 2010.

\begin{abstract}
Hyon, G.-S., Ikeda, K., Hosogi, N., Shinogi, T., and Park, P. 2010. Inhibitory effects of antioxidant reagent in reactive oxygen species generation and penetration of appressoria of Alternaria alternata Japanese pear pathotype. Phytopathology 100:840-847.

In the Japanese pear pathotype of Alternaria alternata, $\mathrm{H}_{2} \mathrm{O}_{2}$ is generated solely from penetration pegs and not from other portions of subcuticular hyphae within the pectin layers of host leaves. A close association between $\mathrm{H}_{2} \mathrm{O}_{2}$ generation and fungal aggressiveness is expected because the pegs are important for fungal penetration into the

host epidermis. To determine the potential role of reactive oxygen species in microbial pathogenicity, we studied the inhibitory effects of the antioxidant reagent ascorbic acid and the NADPH oxidase inhibitor diphenylene iodonium on infection of the pathogen. In our study, we showed $\mathrm{H}_{2} \mathrm{O}_{2}$ generation to be inhibited by inoculation with the mixture of ascorbic acid or diphenylene iodonium and spores at the pegs in the spore-inoculated host leaves. The decrease of generation in the pegs resulted in penetration failure, indicating that $\mathrm{H}_{2} \mathrm{O}_{2}$ generation probably contributed to strengthening of the penetration and probably was recruited by NADPH oxidase.
\end{abstract}

Alternaria alternata (Fr.) Keissl. is a ubiquitous saprophytic fungus that can be found in soil and rotting plant tissues worldwide (24). Some strains are well known to cause severe plant diseases $(19,31)$. The pathogenicity of some fungi has been determined by the production of distinctive host-specific toxins (HSTs) from each of the A. alternata pathotypes, which are capable of causing lesions similar to the ones caused by the pathotypes in some plant diseases $(18,31)$. Among the A. alternata pathotypes, the Japanese pear pathotype is the causal fungus of black spot disease in Japanese pear plants (Pyrus pyrifolia var. culta) (29) and produces two different HSTs: AK-toxins I and II (18). It has been widely accepted that AK-toxins are compatible with the disease determinants of pathogenicity, which have exhibited host specificity in some cultivars of pear plants (23). The toxins induced the characteristic veinal necrosis only in sensitive pear plants such as pear cv. Nijisseiki $(17,23)$. The toxins produced by the $A$. alternata Japanese pear pathotype caused cell death in the host plants after treatment. The toxins produced by A. alternata are capable of inducing plant disease in sensitive plants but not in insensitive plants. In addition, fungi that do not produce these toxins cannot even parasitize the host. These results suggested that the toxins played a central role for suppression of host defense responses.

Recently, the importance of pathogenesis-related reactive oxygen species (ROS) has been widely accepted in some plantmicrobe interactions $(6-9,13,25,30)$. It has also been reported that ROS acts as a toxic agent for the induction of host cell death $(6,7,13,30)$. ROS has been recognized to be catalyzed by fungal NADPH oxidase (Nox). ROS production has been reported to be involved in the appressoria formation of Magnaporthe grisea (8).

Corresponding author: P. Park; E-mail address: ppark@kobe-u.ac.jp

doi:10.1094/PHYTO-100-9-0840

(c) 2010 The American Phytopathological Society
In another fungus, Claviceps purpurea, the deletion of nox 1 influenced three different events: namely, spore germination, resistance to oxidative stress, and virulence (9). In Botrytis cinerea, almost all noxA/noxB double mutants became completely nonpathogenic to the host plants (25). ROS was also generated from both plasma membranes and cell walls in the pegs of the A. alternata Japanese pear pathotype but not from other portions such as the subcuticular hyphae within host pectin layers (27). We expected a close association of ROS generation with fungal aggressiveness because the pegs were special infection structures for fungal penetration into the host epidermis. Our results suggested that ROS generation was associated with the early penetration stage of host cell walls, especially peg aggressiveness. The association between ROS generation and aggressiveness has remained unclear until now. In the context of this hypothesis, we studied the effects of the antioxidant ascorbic acid (AA) on A. alternata pathogenicity and whether ROS generation was related to fungal aggressiveness of the A. alternata Japanese pear pathotype. AA is well known as a scavenger of peroxyl radicals (3), superoxide radicals (1), and singlet oxygens (14). Furthermore, to determine the relationship between ROS generation and Nox in the infection structures of the pathotype, we studied the effects of the Nox inhibitor diphenylene iodonium (DPI) (20). Thus, the aim of this study was to examine the inhibitory effects of AA and DPI on infection of the pathogen, in order to determine the potential role of ROS in microbial pathogenicity.

\section{MATERIALS AND METHODS}

Plants and pathogen. We used two different cultivars of Japanese pear plants ( $P$. pyrifolia var. culta) in this study. $\mathrm{Cv}$. Nijisseiki is susceptible to the A. alternata Japanese pear pathotype, whereas cv. Chojuro is resistant to it. The meristems of Japanese pear shoot tips or axillary buds were obtained from both 
cultivars and were grown by an in vitro propagation method described by Banno et al. (2). We used a woody plant medium (15) to propagate the buds. The shoots were placed at $25^{\circ} \mathrm{C}$ during a 12-h photoperiod. We used the A. alternata Japanese pear pathotype O-276 as the fungal isolate, which was provided by the Laboratory of Plant Pathology of Tottori University in Japan. We preserved the isolate on potato dextrose agar (PDA) slants.

Spore inoculation and antioxidant reagent treatment. We prepared the fungal spores by the method modified by Murakami et al. (16). Mycelial plugs of the isolate were placed on an oatmeal agar medium (40 g of oatmeal, $20 \mathrm{~g}$ of agar, and $5 \mathrm{~g}$ of sucrose in 1 liter of water) in a petri dish and incubated at $25^{\circ} \mathrm{C}$ for 5 days. We rubbed the surfaces of the aerial mycelia with a spatula and incubated the rubbed mycelia at $25^{\circ} \mathrm{C}$ for 3 days under continuous fluorescent illumination (FL20S BLB 20W; Toshiba Corp., Tokyo). After illumination, we added sterile distilled water to the mycelial mats and rubbed the mats with a spatula. The spore suspension was then filtered using Kimwipe paper (Crecia Corp., Tokyo). Fluids were centrifuged once at 3,000 rpm for $5 \mathrm{~min}$. We collected the spores and suspended them in distilled water as a control and adjusted the spore concentration to $5 \times 10^{5}$ spores $/ \mathrm{ml}$ for lesion observation. We adjusted some spore suspensions to the same spore concentration and added AA (Wako, Osaka, Japan) at concentrations of 1, 10, and $100 \mathrm{mM}$ to some of the suspensions. A DPI (Sigma-Aldrich, St. Louis) was dissolved in dimethyl sulfoxide and sterile distilled water and employed at final concentrations of $1 \mu \mathrm{M}$. Then, we sprayed the spore suspensions onto the surfaces of susceptible, resistant, and heat-shocked resistant leaves. The heat-shocked resistant shoots were prepared by the method described by Shinogi et al. (27). For heat treatment, we poured sterilized water at $55^{\circ} \mathrm{C}$ into growth boxes for $5 \mathrm{~s}$, and the plants in the box were incubated for $5 \mathrm{~s}$ in the water. After removing water from the boxes, we turned the boxes upside down and placed them on filter paper at room temperature to remove the water drops from the plant surfaces. Within $30 \mathrm{~min}$ after the heat treatment, spores were inoculated into the heat-shocked leaves. For light microscopy, drops $(20 \mu \mathrm{l})$ of the suspension were placed both on the cellulose membranes and on the upper epidermis of susceptible, resistant, and heatshocked resistant leaves. For electron microscopy, drops $(20 \mu \mathrm{l})$ of the spore suspensions with a concentration of $5 \times 10^{6}$ spores $/ \mathrm{ml}$ were placed on the upper epidermis of susceptible, resistant, and heat-shocked leaves. The spore-inoculated shoots were grown at $25^{\circ} \mathrm{C}$ during a 12 -h photoperiod.

Cytological assays. After the inoculation, the spore-inoculated leaves were detached from the shoots and treated with a mixture of ethanol and acetic acid (96:4, vol/vol) for removing chlorophyll at $25^{\circ} \mathrm{C}$ overnight. The specimens were stained with $0.25 \%$ Coomassie brilliant blue (Nakarai, Tokyo) in a mixture of methanol, acetic acid, and distilled water (50:5:45, vol/vol/vol). We observed the infection behavior of the pathotype in the stained specimens with an Olympus BX51 microscope (Olympus, Tokyo).

Cytochemistry for hydrogen peroxide. We used a modified cytochemical method for $\mathrm{H}_{2} \mathrm{O}_{2}$ detection $(4,5,27)$. Leaf pieces ( 2 by $3 \mathrm{~mm}$ ) of the inoculated susceptible, resistant, and heatshocked resistant leaves were vacuum-infiltrated in $5 \mathrm{mM} \mathrm{CeCl}_{3}$ solution buffered with $50 \mathrm{mM} 3$-( $\mathrm{N}$-morpholino)propanesulfonic acid (MOPS; $\mathrm{pH}$ 7.2) at room temperature for $1 \mathrm{~h}$. The pieces were then incubated in $\mathrm{CeCl}_{3}$ solution at room temperature for $1 \mathrm{~h}$. Some pieces were infiltrated in vacuo only in MOPS without $\mathrm{CeCl}_{3}$ as a control. The pieces were prefixed in $2.5 \%$ glutaraldehyde buffered with $0.1 \mathrm{M}$ cacodylate buffer $\left(\mathrm{pH} \mathrm{7.2)}\right.$ at $4^{\circ} \mathrm{C}$ overnight, then washed with the same buffer three times for 10 min each and postfixed with the $1 \%$ buffered osmium tetroxide at $4{ }^{\circ} \mathrm{C}$ for $1 \mathrm{~h}$. The pieces were then dehydrated in ethanol and embedded in Spurr resin mixture (Nissin EM, Tokyo). Sections with a thickness of 90 to $120 \mathrm{~nm}$ were cut from resin blocks with an ultramicrotome (MT-1; Sorval, Norwalk, CT) by using a diamond knife (Diatome, Binene, Switzerland). Unstained sections were observed with a Hitachi 7100 electron microscope (Hitachi, Hitachinaka, Japan).

Image analysis. We observed each of three different blocks from the leaf pieces of the inoculated susceptible, resistant, and heat-shocked resistant leaves with an electron microscope. The phloem cells of each specimen were photographed at random at the magnification of $\times 5,000$. Electron microscopic negatives were printed in the same typed printing papers at the same magnification. Each of the micrographs was scanned at $600 \mathrm{dpi}$ (dots per inch) and saved as a .jpg file. We analyzed the volume of cerium-reactive products on the electron micrographs by using the image analysis software Image $\mathrm{J}$ (http://rsb.info.nih.gov/ij/ index.html). We then measured the areas of cerium-reactive products in appressorial cell walls. The relative ratio of the product area per unit area $\left(\mu \mathrm{m}^{2}\right)$ of appressoria-cell wall was determined as the ratio of area of $\mathrm{CeCl}_{2}$ deposit $\left(\mu \mathrm{m}^{2}\right) /$ area of appressoria-cell wall $\left(\mu \mathrm{m}^{2}\right)$.

Assay for AK-toxin production. We examined whether AKtoxin I was produced from the germinating spore by the method described by Hayashi et al. (11). We uniformly sprinkled $100-\mathrm{ml}$ spore suspensions $\left(5 \times 10^{5}\right.$ spores $\left./ \mathrm{ml}\right)$ onto paper towels and incubated them in a moist chamber for $24 \mathrm{~h}$ at $25^{\circ} \mathrm{C}$. We measured the formation rate of the germinated spores in the time-course under a light microscope. The spore-germination fluids were harvested by squeezing the towels and filtering the liquid through a filter paper to remove the spores. The filtrates were adjusted to $\mathrm{pH} 5.5$ with $1 \mathrm{M} \mathrm{KH}_{2} \mathrm{PO}_{4}$ and the toxin was extracted with diethyl ether. After removal of the solvent by vacuum evaporation, the residues were dissolved in $1 \mathrm{ml}$ of methanol and the samples were subjected to a high-pressure liquid chromatography (HPLC) model of the Shimadzu Corp. (SCL-10A, SPD-10A, LC-10AS; Kyoto, Japan). The stainless steel HPLC column $(4.6 \times 250 \mathrm{~mm})$ was packed in the reverse-phase Shim-pack CLC-ODS M (Shimadzu Corp., Kyoto, Japan). HPLC-grade solvents were used for analysis. The elutions were made with a mixture of acetonitrile, acetic acid, and water $(50: 1: 49, \mathrm{vol} / \mathrm{vol} / \mathrm{vol})$ at a flow rate of $1 \mathrm{ml} / \mathrm{min}$, and detection of the toxin was carried out by measuring absorbance at $290 \mathrm{~nm}$. Crystalline AK-toxins I and II, which were used as the authentic toxin in this study, were provided by Professor $\mathrm{H}$. Miyagawa of the Division of Applied Life Sciences, Graduate School of Agriculture, Kyoto University, Japan.

\section{RESULTS}

Inhibitory effects of AA on the formation of infection structures of the A. alternata Japanese pear pathotype. We examined the effects of an antioxidant agent, AA, on the formation of the germ tubes, appressoria, and infection hyphae on a cellulose membrane to determine the potential role of ROS, germinated in the infection structures, in pathogenicity. When $A$. alternata spore suspensions were incubated at $25^{\circ} \mathrm{C}$ for $24 \mathrm{~h}$ on the membranes, most of the spores germinated in the water, appressoria were formed in half of the germinated spores, and infection hyphae were produced from half of the appressoria (Fig. $1 \mathrm{~A}$ and $\mathrm{B})$. The antioxidant agent of $1 \mathrm{mM}$ AA inhibited only the formation of infection hyphae (Fig. 1A and C), while the 10- and $100-\mathrm{mM}$ agents suppressed only the formation of appressoria and infection hyphae (Fig. 1A, D, and E). In contrast, agents with concentrations of 1,10 , and $100 \mathrm{mM}$ caused no germ tube formation (Fig. 1A, C, D, and E). From the results obtained, $1 \mathrm{mM}$ AA is the most suitable concentration for understanding the role of AA in fungal invasion. Therefore, we used $1 \mathrm{mM}$ AA in our experiments.

Suppressive effects of AA on lesion formation in Japanese pear leaves inoculated with the A. alternata pathotype spores. We examined lesion formation in the pear leaves inoculated with A. alternata pathotype spores to understand the role of AA in 
plant infection. The spores caused typical black spot lesions in susceptible leaves $24 \mathrm{~h}$ after inoculation (Fig. 2A) but not in resistant ones (Fig. 2B). Susceptibility was induced in the heatshocked leaves of resistant shoots, after which they were inoculated with spores for $24 \mathrm{~h}$ (Fig. 2C). These events have already been reported by Otani et al. $(21,22)$. We observed lesions in both the susceptible and heat-shocked resistant leaves $24 \mathrm{~h}$ after spore inoculation. On the other hand, pretreatment with AA caused a decrease of lesions in both susceptible and heat-shocked resistant leaves, followed by postinoculation with spores (Fig. 2D, F, and G). We did not observe any lesions in the inoculated resistant leaves regardless of whether or not AA treatment was performed (Fig. 2B, E, and G).

A
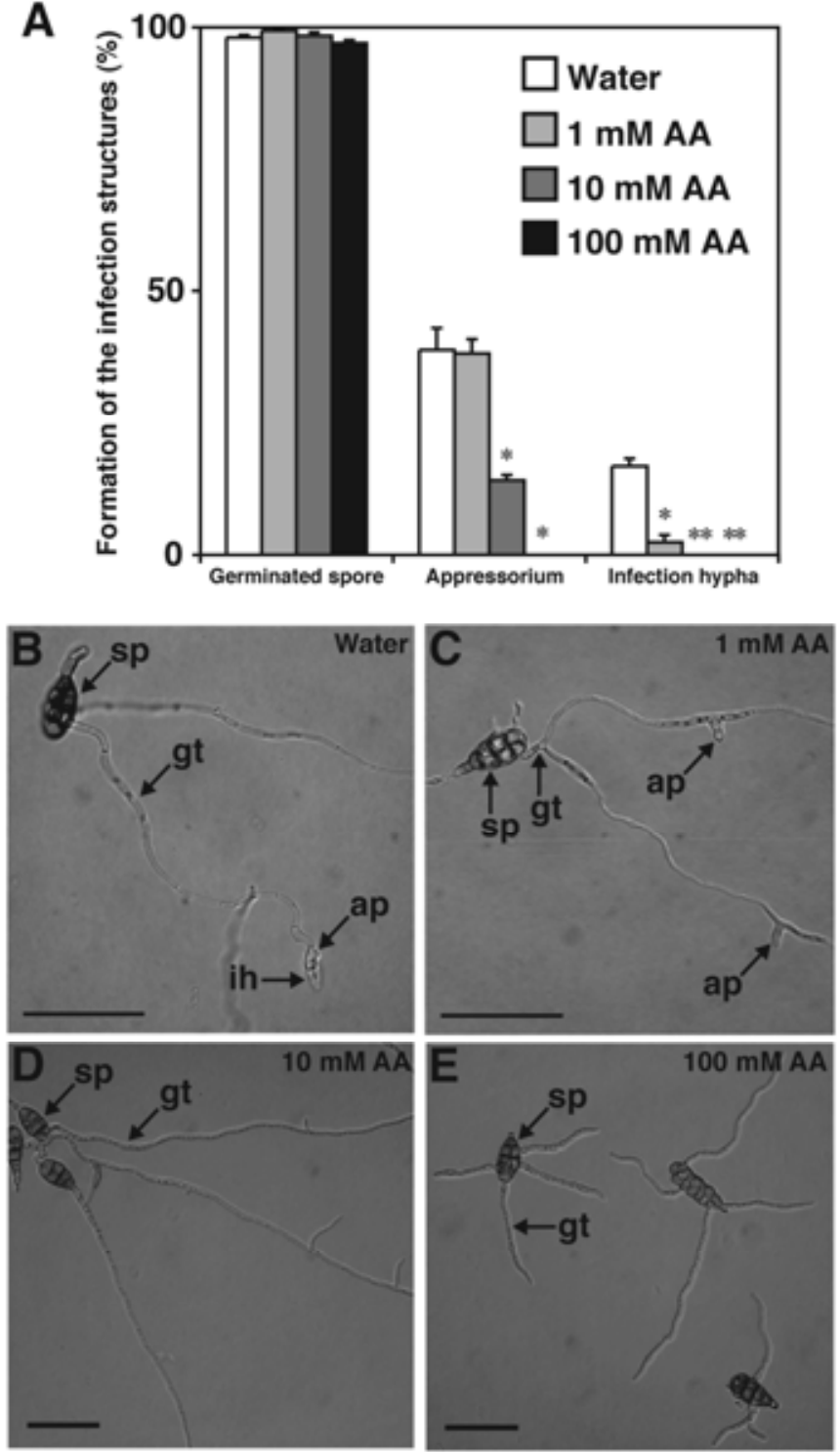

Fig. 1. Formation of infection structures of Alternaria alternata Japanese pear pathotype on cellulose membrane in the presence of 1,10 , or $100 \mathrm{mM}$ ascorbic acid (AA) or in its absence at $25^{\circ} \mathrm{C}$ for $24 \mathrm{~h}$. Abbreviations: $\mathrm{sp}=$ spore; gt = germ tube; ap = appressorium; ih = infection hypha. Bars $=25 \mu \mathrm{m}$. $\mathbf{A}$ and $\mathbf{B}$, Spores were usually germinated in the water, appressoria were formed in half of the germinated spores, and infection hyphae were produced in half of the appressoria. A and C, Germ tubes and appressoria were well formed even in the presence of $1 \mathrm{mM}$ AA but infection hyphae were not. A, D, and $\mathbf{E}$, In contrast, germ tubes were well formed in the presence of both 10 and $100 \mathrm{mM} \mathrm{AA}$ but infection hyphae were not. Note that, with treatment of $100 \mathrm{mM}$ AA (E), neither appressoria nor infection hyphae were observed. Averages and standard errors (SEs) from three independent experiments are shown. Bars indicate SE; * and ** indicate $P<0.05$ and 0.01 , respectively, versus water treatment.
Inhibitory effects of AA on the formation of infection hyphae of the $A$. alternata pathotype in pear leaves inoculated with the spores. We microscopically observed the differentiation of A. alternata infection hyphae in susceptible, resistant, and
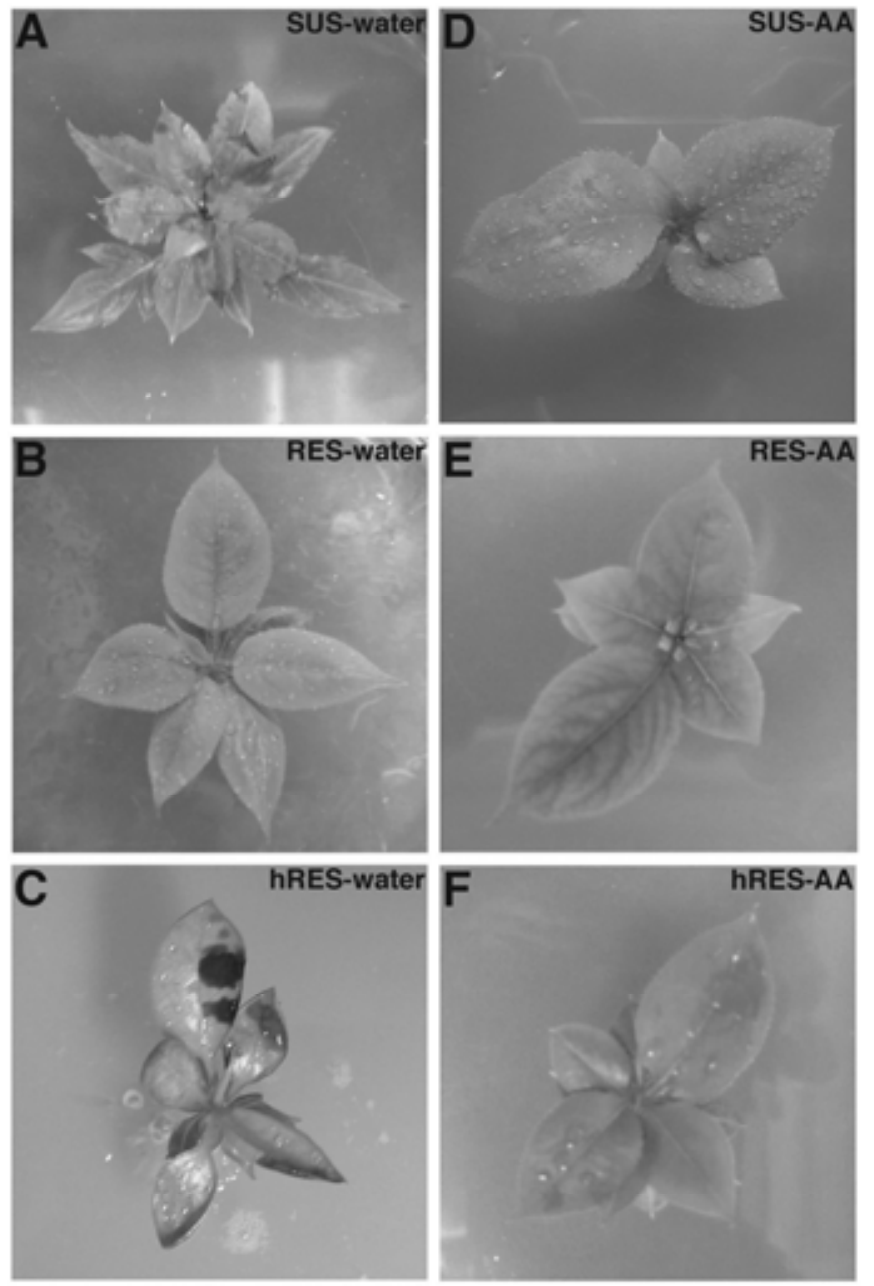

G

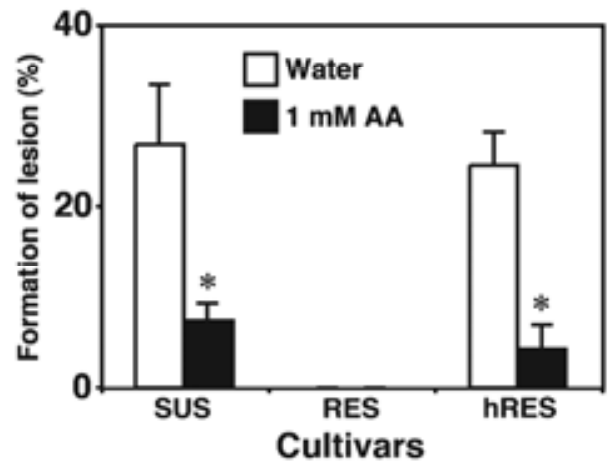

Fig. 2. Suppressive effects of ascorbic acid (AA) on lesion formation of susceptible, resistant, and heat-shocked resistant leaves inoculated with Alternaria alternata Japanese pear pathotype spores $\left(5 \times 10^{5} \mathrm{spores} / \mathrm{ml}\right)$ at $25^{\circ} \mathrm{C}$ for $24 \mathrm{~h}$ in the $\mathbf{A}$ to $\mathbf{C}$, absence or $\mathbf{D}$ to $\mathbf{F}$, presence of $1 \mathrm{mM} \mathrm{AA}$. $\mathbf{G}$, Relative area ratios of lesion and lesion area per unit area of pear leaves were examined in both column and standard errors (SEs). Abbreviations: SUS = susceptible leaves; RES = resistant leaves; hRES = heat-shocked resistant leaves. Bars indicate the SE of the mean $(n=10)$ and * indicates $P<0.01$ versus water treatment. Black spot lesions were observed in $\mathbf{A}$ and $\mathbf{G}$, susceptible and $\mathbf{C}$ and $\mathbf{G}$, heat-shocked resistant leaves $24 \mathrm{~h}$ after the inoculation with the spore suspensions but not in the $\mathbf{B}$ and $\mathbf{G}$, inoculated resistant leaves. On the other hand, lesion areas decreased in $\mathbf{D}$ and $\mathbf{G}$, susceptible and $\mathbf{F}$ and $\mathbf{G}$, heat-shocked resistant leaves inoculated with the mixture of spores and AA. 
heat-shocked resistant leaves (Fig. 3A to G). Infection hyphae were produced from appressoria within 6 to $12 \mathrm{~h}$ after spore inoculation in susceptible leaves, whereas they formed more frequently within $6 \mathrm{~h}$ after inoculation in heat-shocked resistant leaves. However, the infection hyphae were not usually produced from appressoria in resistant leaves inoculated with the spores (Fig. 3C). Elongated infection hyphae were formed from appressoria in susceptible leaves $24 \mathrm{~h}$ after inoculation (Fig. 3B) but not in resistant leaves, although secondary hyphae were sometimes produced from the same appressoria (Fig. 3C). In heat-shocked resistant leaves, the same infection hyphae formed from appressoria as in the spore-inoculated susceptible leaves (Fig. 3D). In addition, the formation rate of infection hyphae was higher in the heat-shocked leaves than in the susceptible ones (Fig. 3A). In the presence of AA, infection hyphae were produced neither in the susceptible leaves $24 \mathrm{~h}$ after inoculation (Fig. 3A and E) nor in the heat-shocked resistant leaves $12 \mathrm{~h}$ after inoculation. The suppressive effect of $1 \mathrm{mM} \mathrm{AA}$ on infection hyphae formation was recognized in heat-shocked resistant leaves even $72 \mathrm{~h}$ after inoculation (Fig. 3A and G). This indicated that pretreatment with the antioxidant reagent inhibited fungal penetration in susceptible and heat-shocked resistant leaves after spore inoculation.

Inhibitory effects of $\mathrm{AA}$ on $\mathrm{H}_{2} \mathrm{O}_{2}$ generation of $A$. alternata infection structures. $\mathrm{H}_{2} \mathrm{O}_{2}$ has previously been detected ultrastructurally by a cytochemical method using cerium chloride (27). When $\mathrm{H}_{2} \mathrm{O}_{2}$ molecules generated from fungi or plants react with exogenous cerium ions, insoluble cerium perhydroxides are quickly formed and precipitated in reaction sites in situ. Cerium perhydroxides were revealed as electron-dense products under an electron microscope because they involve a heavy metal. Germ tubes were formed from most of the spores on the plant surfaces $6 \mathrm{~h}$ after inoculation. The swollen tips of germ tubes produced appressoria from which penetration pegs emerged. An extracellular matrix appeared to be produced from the appressoria and was located between the appressoria and plant cuticles (Fig. 4A to C). Later, the pegs invaded the pectin layers of the pear leaves through cuticular penetration and then changed into thick, subcuticular hyphae. We observed $\mathrm{H}_{2} \mathrm{O}_{2}$ generation in the appressorial bottoms, pegs, and necks of the subcuticular hyphae in the pectin layers of the spore-inoculated pear leaves (Fig. 4A to C) but not in the host plants. The $\mathrm{H}_{2} \mathrm{O}_{2}$-generated sites were spatially compatible with the cell walls and plasma membranes of the appressoria, pegs, and necks in the inoculated susceptible, resistant, and heat-shocked resistant leaves. We observed a large volume of $\mathrm{H}_{2} \mathrm{O}_{2}$-reactive products at fungal infection structures in the interaction between susceptible leaves and the pathotype and between heat-shocked resistant leaves and the pathotype (Fig. 4A and $\mathrm{C}$ ), whereas we observed a small volume of the products in the interaction between resistant leaves and pathotype (Fig. 4B).

The 24-h treatment of AA with the spore suspension caused no $\mathrm{H}_{2} \mathrm{O}_{2}$ generation at fungal infection structures in the sporeinoculated susceptible (Fig. 4D), resistant (Fig. 4E), and heatshocked resistant leaves (Fig. 4F). $\mathrm{H}_{2} \mathrm{O}_{2}$ generation was not generally recognized at fungal infection structures even with an increased treatment time (Fig. 4G). We did not observe $\mathrm{H}_{2} \mathrm{O}_{2}$ reactive products in the controls of MOPS solution without $\mathrm{CeCl}_{3}$; in fact, the infection structures did not show $\mathrm{H}_{2} \mathrm{O}_{2}$-reactive products in the spore-inoculated susceptible (Fig. 4H), resistant (Fig. 4I), and heat-shocked resistant leaves (Fig. 4J). Treatment with AA caused no fungal invasion of the pathotype even in the spore-inoculated susceptible and heat-shocked resistant leaves with suppressive $\mathrm{H}_{2} \mathrm{O}_{2}$ generation (Fig. 4D and F).

Suppressive effects of DPI in penetration and ROS generation of A. alternata pathotype appressoria. To understand the effects of DPI on plant infection, we examined lesion formation in pear leaves inoculated with A. alternata pathotype spores to understand the role of DPI in plant infection (Fig. 5A to D). We observed lesions in both the susceptible and heat-shocked
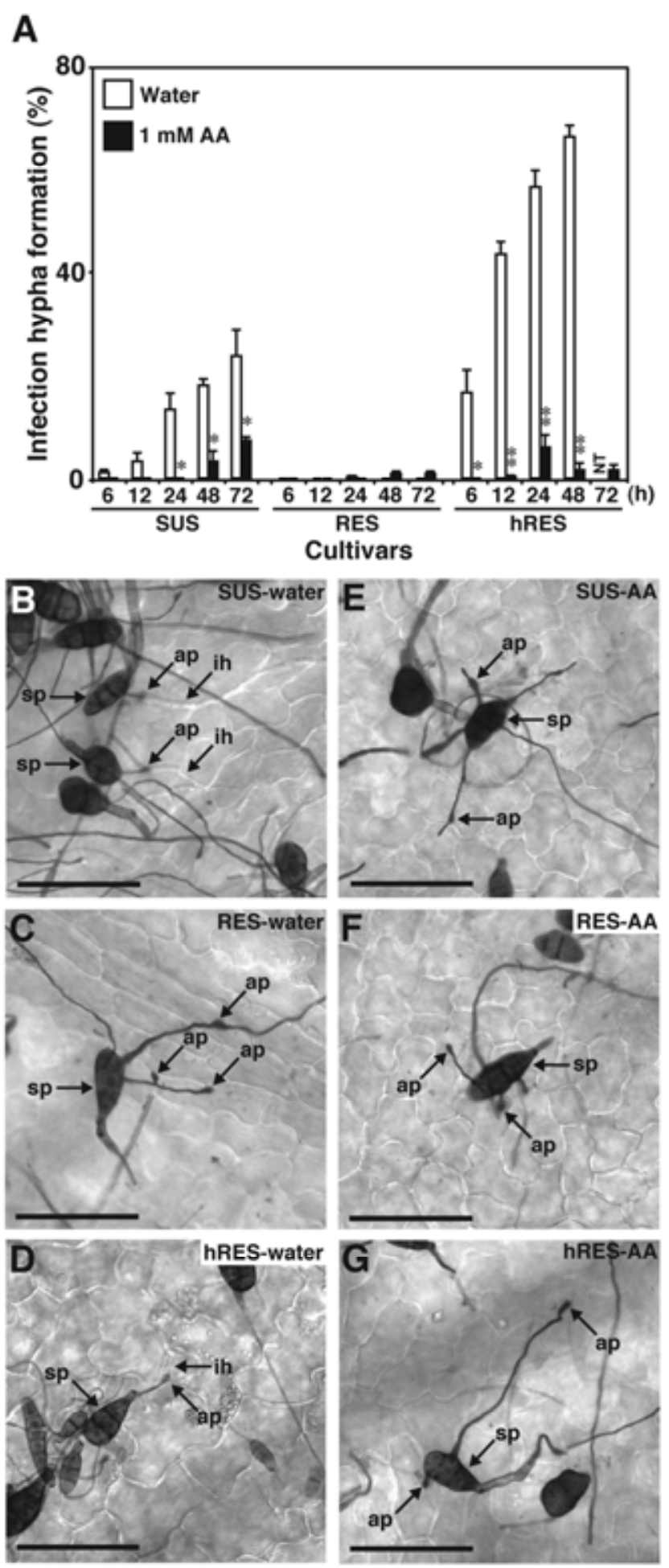

Fig. 3. Inhibitory effects of $1 \mathrm{mM}$ ascorbic acid (AA) on Alternaria alternata infection hyphae formation in susceptible, resistant, and heat-shocked resistant leaves inoculated with the pathotype spores $\left(5 \times 10^{5}\right.$ spores $\left./ \mathrm{ml}\right)$ at $25^{\circ} \mathrm{C}$ for 24 h. A, Formation rate of infection hyphae on pear leaves inoculated with spores in the presence or absence of $1 \mathrm{mM}$ AA. Abbreviations: SUS = susceptible leaves; RES = resistant leaves; hRES = heat-shocked resistant leaves. Average and standard errors (SEs) of three independent experiments are shown. Bars indicate SE; NT $=$ not tested; $*$ and $* *$ indicate $P<0.05$ and 0.01 , respectively, versus water treatment. Infection hyphae were seen in $\mathbf{B}$, inoculated susceptible and $\mathbf{D}$, inoculated heat-shocked resistant leaves in the absence of AA but not in $\mathbf{C}$, the inoculated resistant leaves. In the presence of AA, formation of infection hyphae was suppressed in $\mathbf{E}$, inoculated susceptible; F, inoculated resistant; and $\mathbf{G}$, inoculated heat-shocked resistant leaves. The inhibitory effect of AA on infection hypha formation was recognized even $72 \mathrm{~h}$ after inoculation (A). Abbreviations: $\mathrm{sp}=$ spore; $\mathrm{gt}=$ germ tube; $\mathrm{ap}=$ appressorium; ih = infection hypha. Bars $=25 \mu \mathrm{m}(\mathbf{B}$ to $\mathbf{F})$. 
resistant leaves $48 \mathrm{~h}$ after inoculation with the spore suspension (Fig. 5A and B). On the other hand, lesion formation was suppressed in both susceptible and heat-shocked resistant leaves that had been inoculated with a mixture of spores and DPI (Fig. 5C and D). An ultramicroscope was used to observe fungal penetration and $\mathrm{H}_{2} \mathrm{O}_{2}$ generation in susceptible and heat-shocked resistant leaves $48 \mathrm{~h}$ after inoculation with pathotype spores in the presence of DPI (Fig. 6A to F). DPI treatment suppressed not only fungal penetration of the pathotype in spore-inoculated susceptible and heat-shocked resistant leaves (Fig. 6C, D, and E) but also $\mathrm{H}_{2} \mathrm{O}_{2}$ generation in these leaves (Fig. 6C, D, and F). These results indicated that pretreatment with the NADPH oxidase inhibitor DPI inhibited both fungal penetration and $\mathrm{H}_{2} \mathrm{O}_{2}$ generation in susceptible and heat-shocked resistant leaves after spore inoculation with the spore suspension.

AK-toxin production. To assess whether treatment with AA and DPI affected AK-toxin production during plant infection, we measured the toxin production by HPLC. HPLC analysis of authentic AK-toxin revealed two separate peaks of AK-toxins I and II that had different retention times (Fig. 7). Both toxins were present in the germination fluids formed in the presence of $1 \mathrm{mM}$ AA or $1 \mu \mathrm{M}$ DPI (Table 1).

\section{DISCUSSION}

In this study, we demonstrated inhibitory effects of the antioxidant AA on the formation of infection structures in the $A$. alternata Japanese pear pathotype on susceptible and heatshocked resistant leaves and cellulose membranes. Although AA at a concentration of $1 \mathrm{mM}$ caused the normal formation of both germ tubes and appressoria in addition to the normal production of AK-toxin, infection hyphae were not formed by the treatment, which probably influenced the suppression of plant disease. This suggests that the inhibition of ROS generation by AA was
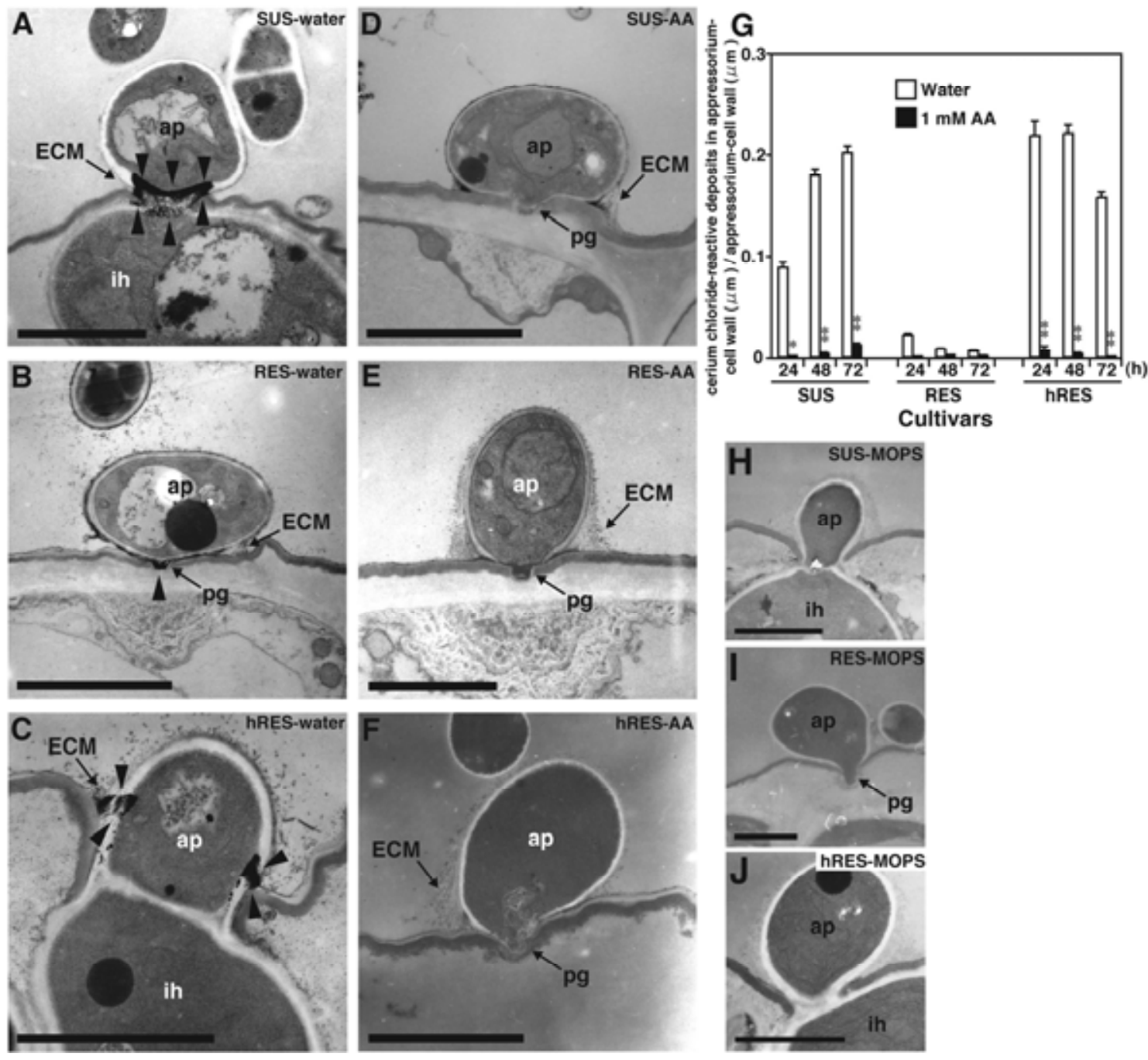

Fig. 4. A to F, Ultrastructural localization of $\mathrm{H}_{2} \mathrm{O}_{2}$ generation and relative area ratio of $\mathrm{H}_{2} \mathrm{O}_{2}$-reactive products in appressoria and penetration pegs of Alternaria alternata Japanese pear pathotype on susceptible, resistant, and heat-shocked resistant leaves inoculated with spores $\left(5 \times 10^{6} \mathrm{spores} / \mathrm{ml}\right)$ at $25^{\circ} \mathrm{C}$ for $24 \mathrm{~h}$ with $1 \mathrm{mM}$ ascorbic acid (AA) or without AA treatment. Abbreviations: SUS = susceptible leaves; RES = resistant leaves; hRES = heat-shocked resistant leaves; ap = appressorium; $\mathrm{pg}=$ penetration peg; ih = infection hypha; $\mathrm{ECM}=$ extracellular matrix. Arrowheads indicate the $\mathrm{H}_{2} \mathrm{O}_{2}$-reactive products. Bars $=5 \mu \mathrm{m}(\mathbf{A}$ to $\mathbf{F}$ and $\mathbf{H}$ to $\mathbf{J})$. G, Relative area ratio of $\mathrm{H}_{2} \mathrm{O}_{2}$-reactive products in appressorium cell wall $\left(\mu \mathrm{m}^{2}\right)$ to appressorium cell wall $\left(\mu \mathrm{m}^{2}\right)$ was measured by image analysis. Averages and standard errors (SEs) of three independent experiments are shown. Bars indicate SE; * and ** indicate $P<0.05$ and 0.01 , respectively, versus water treatment. $\mathrm{H}_{2} \mathrm{O}_{2}$ generation was observed in the appressorial bottoms, pegs, and necks of subcuticular hyphae of the spore-inoculated A, susceptible; B, resistant; and $\mathbf{C}$, heat-shocked resistant pear leaves. A large volume of $\mathrm{H}_{2} \mathrm{O}_{2}$-reactive products was observed at fungal infection structures in the interaction between susceptible leaves and the pathotype and in the interaction between heat-shocked resistant leaves and the pathotype (A, C, and G); however, a small volume of the products was observed in the interaction between resistant leaves and pathotype (B and G). Twenty-four-hour treatment of AA with the spore suspension caused no $\mathrm{H}_{2} \mathrm{O}_{2}$ generation at fungal infection structures in the spore-inoculated $\mathbf{D}$ and $\mathbf{G}$, susceptible; $\mathbf{E}$ and $\mathbf{G}$, resistant; and $\mathbf{F}$ and $\mathbf{G}$, heat-shocked resistant leaves. $\mathrm{H}_{2} \mathrm{O}_{2}$-reactive products were not observed at the infection structures in the spore-inoculated $\mathbf{H}$, susceptible; I, resistant; or $\mathbf{J}$, heat-shocked resistant leaves as a control when the inoculated leaves were exposed to 3-( $N$-morpholino)propanesulfonic acid (MOPS) solution without $\mathrm{CeCl}_{3}$. 
associated with the loss of one of the pathogenicity factors, although the pathogenicity mechanism remains unclear.

Shinogi et al. (27) reported that $\mathrm{H}_{2} \mathrm{O}_{2}$ was generated at the appressorial bottoms or penetration pegs: when the spore suspensions were inoculated in Japanese pear leaves, a large volume of $\mathrm{H}_{2} \mathrm{O}_{2}$ generation was produced at the pegs involving appressorial bottoms in spore-inoculated susceptible and heat-shocked resistant leaves whereas a small volume was produced at those in the inoculated resistant leaves. In the present study, $\mathrm{H}_{2} \mathrm{O}_{2}$ generation was completely inhibited by inoculation with the mixture of $1 \mathrm{mM}$ AA and spores at the pegs and appressorial bottoms in the spore-inoculated susceptible, resistant, and heat-shocked resistant leaves. This indicates that the antioxidant treatment inhibited ROS generation of the appressoria or pegs in the inoculated pear leaves. This inhibition in the pegs resulted in the failure of penetration, suggesting that $\mathrm{H}_{2} \mathrm{O}_{2}$ generation contributed to strengthening of the peg penetration.

There is limited available information about resistance mechanisms in the interaction between the pathotype and pear plants. Antifungal compounds such as phytoalexins have not yet been identified in these interactions. Some lines of evidence for the defense substances were provided and an infection-inhibiting factor (IIF)—namely, 3,5-di- $O$-caffeoylquinic acid-was reported (12). IIF was accumulated in the inoculated portions of Japanese pear leaves as the host response by inoculating with nonpathogenic A. alternata. The factor inhibited the formation of infection hyphae although it caused no inhibition of appressorial formation. Pretreatment with spore-germination fluids from various nonpathogenic Japanese pear plants was also reported to reduce infection of the pear pathotype $(10,19,21,28)$. In addition, catechin, which is produced in strawberry leaves, has been known to act as a protective agent against fungal infection in other plants. Catechin is a possible IIF (32): catechin accumulated in the leaves of the host in response to inoculation with non-
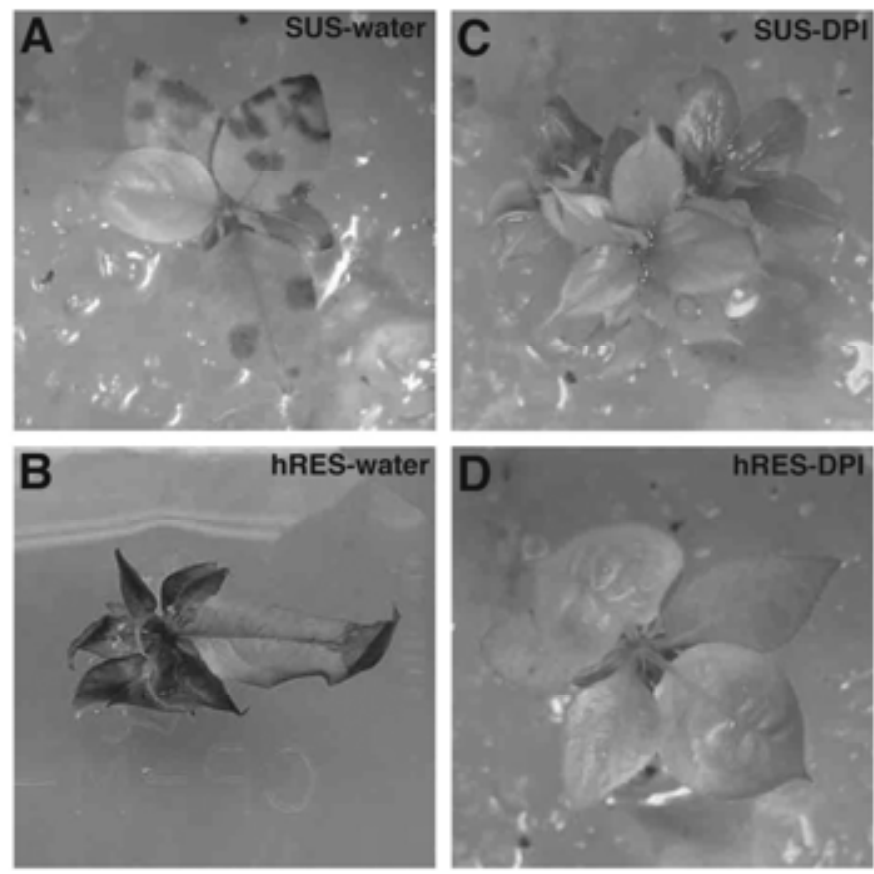

Fig. 5. Suppressive effects of diphenylene iodonium (DPI) on lesion formation in susceptible and heat-shocked resistant leaves inoculated with Alternaria alternata Japanese pear pathotype spores $\left(5 \times 10^{5}\right.$ spores $\left./ \mathrm{ml}\right)$ at $25^{\circ} \mathrm{C}$ for $48 \mathrm{~h}$ in the $\mathbf{A}$ and $\mathbf{B}$, absence or $\mathbf{C}$ and $\mathbf{D}$, presence of $1 \mu \mathrm{M}$ DPI. Abbreviations: SUS = susceptible leaves; hRES = heat-shocked resistant leaves. Black spot lesions were observed in the $\mathbf{A}$, susceptible and $\mathbf{C}$, heat-shocked resistant leaves $48 \mathrm{~h}$ after the inoculation with the spore suspensions. However, the lesion areas decreased in $\mathbf{C}$, susceptible and $\mathbf{D}$, heat-shocked resistant leaves that had been inoculated with a mixture of spores and DPI. pathogenic A. alternata, which resulted in suppressive infection of the strawberry pathotype of A. alternata in spite of AF-toxin production by the pathotype (32). Data on these factors may support our conclusion that antioxidant regents such as AA play
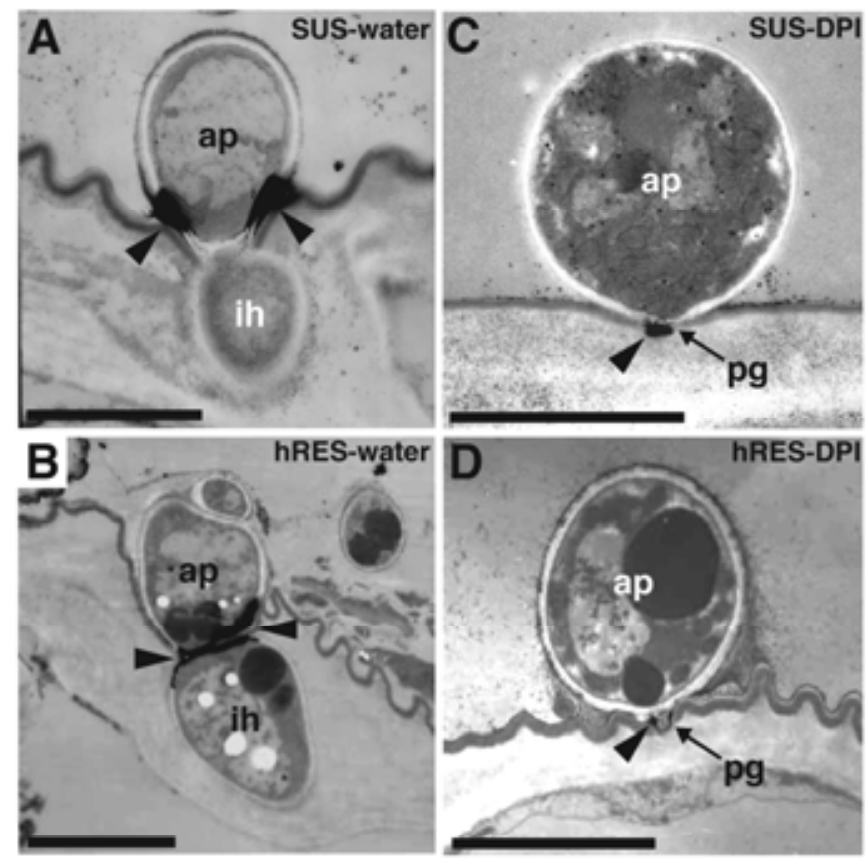

E

$\mathbf{F}$
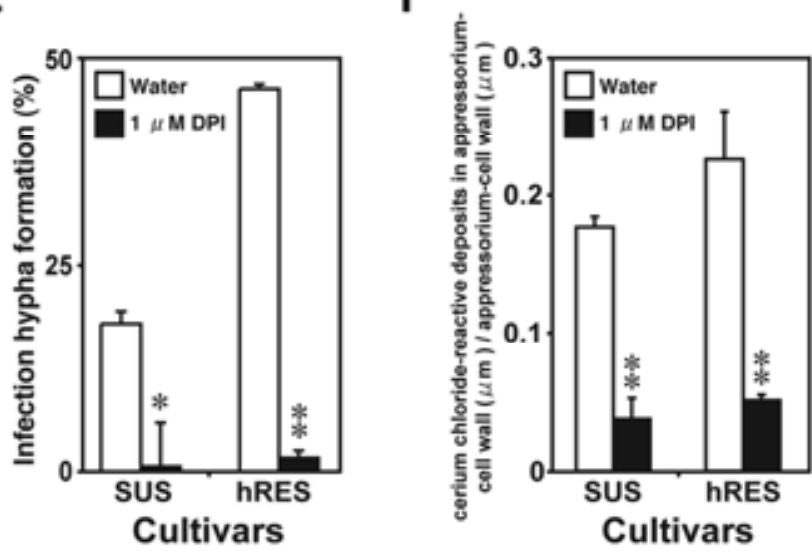

Fig. 6. A to D, Ultrastructural observation of penetration and $\mathrm{H}_{2} \mathrm{O}_{2}$ generation in the appressoria of Alternaria alternata Japanese pear pathotype in susceptible and heat-shocked resistant leaves inoculated with spores $\left(5 \times 10^{6}\right.$ spores $/ \mathrm{ml}$ ) at $25^{\circ} \mathrm{C}$ for $48 \mathrm{~h}$ in the presence or absence of $1 \mu \mathrm{M}$ diphenylene iodonium (DPI). Abbreviations: SUS = susceptible leaves; hRES = heatshocked resistant leaves; ap = appressorium; pg = penetration peg; ih = infection hypha. Arrowheads indicate the $\mathrm{H}_{2} \mathrm{O}_{2}$-reactive products. Bars $=5 \mu \mathrm{m}$ (A to D). E, Formation rate of infection hyphae on pear leaves inoculated with spores in the presence or absence of $1 \mu \mathrm{M}$ DPI. Averages and standard errors (SEs) of three independent experiments are shown. Bars indicate SE; * and ** indicate $P<0.05$ and 0.01 , respectively, versus water treatment. F, Ratio of the area of $\mathrm{H}_{2} \mathrm{O}_{2}$-reactive deposited in the appressorium cell wall $\left(\mu \mathrm{m}^{2}\right)$ to the area of the appressorium cell wall $\left(\mu \mathrm{m}^{2}\right)$ was measured by image analysis. Averages and SE of three independent experiments are shown. Bars indicate SE; ** indicates $P<0.01$ versus water treatment. Infection hyphae were seen in $\mathbf{A}$ and $\mathbf{E}$, inoculated susceptible and $\mathbf{B}$ and $\mathbf{E}$, inoculated heat-shocked resistant leaves in the absence of DPI. In the presence of DPI, the formation of infection hyphae was suppressed in $\mathbf{C}$ and $\mathbf{E}$, inoculated susceptible and $\mathbf{D}$ and $\mathbf{E}$, inoculated heat-shocked resistant leaves. A large volume of $\mathrm{H}_{2} \mathrm{O}_{2}$ reactive products was observed in fungal infection structures during the interaction between susceptible leaves and that between heat-shocked resistant leaves and the pathotype $(\mathbf{A}, \mathbf{B}$, and $\mathbf{F})$; however, a small volume of these products was observed during the interaction between susceptible and heatshocked resistant leaves and the pathotype in the presence of $1 \mu \mathrm{M}$ DPI $(\mathbf{C}, \mathbf{D}$, and $\mathbf{F}$ ). 


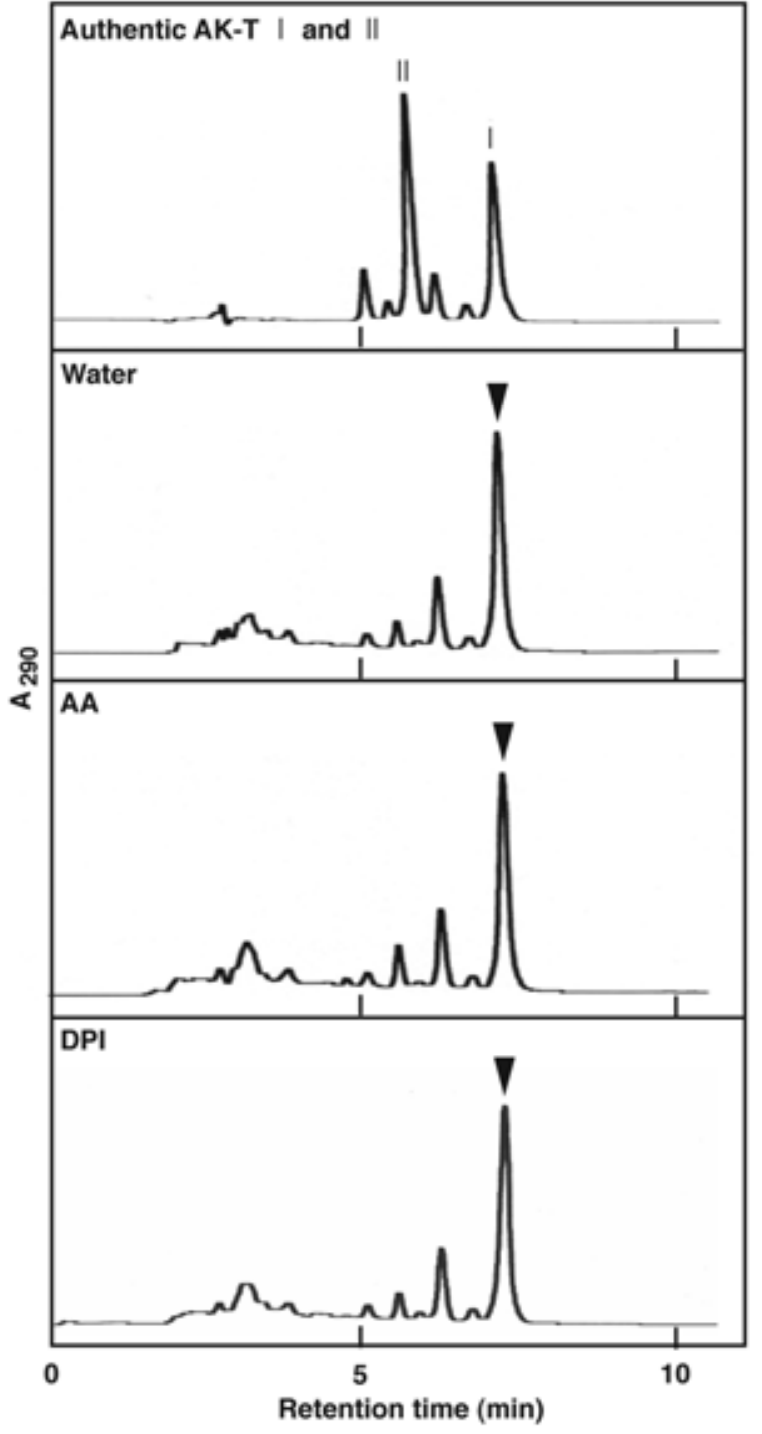

Fig. 7. High-pressure liquid chromatography (HPLC) analysis of the sporegermination fluid of the Alternaria alternata Japanese pear pathotype. Germination fluids that were harvested after incubation for $24 \mathrm{~h}$ in the presence or absence of $1 \mathrm{mM}$ ascorbic acid (AA) or $1 \mu \mathrm{M}$ diphenylene iodonium (DPI) were subjected to HPLC analysis and found to contain AK-toxins I and II. Arrowheads indicate the peaks of toxin production. HPLC analysis detected the same amount of host-specific AK-toxin I in the germination fluids in both the presence and absence of AA and DPI. The AK-toxin I peak had a retention time of 7.5 min under the conditions employed, which corresponded to that of authentic AK-toxin I. AK-toxin II was detected at very low levels in the fluid under all three experimental conditions.

a role in suppressing infection hyphae via decreased ROS generation.

In this study, we showed that ROS generation is probably related to fungal aggressiveness of the A. alternata Japanese pear pathotype, although the mechanism by which fungal aggressiveness is associated with ROS generation remains unclear. However, these are some lines of evidence, and these may help in clarifying the role of ROS in fungal aggressiveness. It has been reported that ROS accumulation is localized in the apical regions of Aspergillus nidulans hyphae and that Nox or related flavoproteins are responsible for ROS accumulation in apical regions and the regulation of apical dominance (26). Our studies showed that DPI, which is a Nox inhibitor, suppresses both $\mathrm{H}_{2} \mathrm{O}_{2}$ generation and fungal penetration in appressoria on susceptible leaves and heat-shocked resistant leaves. Our results also suggested that ROS generation together with Nox activity contribute to the pegmediated invasion in host cell walls.
TABLE 1. Determination of the amount of AK-toxin produced by the pathotype using high-pressure liquid chromatography analysis of the sporegermination fluid in the presence or absence of $1 \mathrm{mM}$ ascorbic acid (AA) and $1 \mu \mathrm{M}$ diphenylene iodonium (DPI) ${ }^{\mathrm{a}}$

\begin{tabular}{lcc}
\hline & \multicolumn{2}{c}{ AK-toxin production } \\
\cline { 2 - 3 } Concentrations & Toxin I & Toxin II \\
\hline Water & $0.051 \pm 0.003$ & ND \\
1 mM AA & $0.048 \pm 0.013$ & ND \\
$1 \mu$ DPI & $0.047 \pm 0.092$ & ND \\
\hline
\end{tabular}

a Amount of each toxin (in femto) released from a single germinated spore. Averages and standard errors of three independent experiments are shown. $\mathrm{ND}=$ not detected

\section{ACKNOWLEDGMENTS}

We thank H. Tabira, of the horticultural station of Tottori Prefecture, Japan, for providing the Japanese pear shoots; H. Otani, Tottori University, Japan, for providing A. alternata Japanese pear pathotype O-276; and T. Tsurushima, Hannan University, Japan, for helping us with the HPLC experiments.

\section{LITERATURE CITED}

1. Aver'yanov, A. A., and Lapikova, P. V. 1988. Fungitoxicity determined by active forms of oxygen in excretions of rice leaves. Fiziol. Rast. (Moscow) 35:1142-1151.

2. Banno, K., Hayashi, S., Tanabe, K., and Tokuzumi, A. 1988. In vitro propagation of Japanese pear rootstocks. Plant Tissue Cult. Lett. 5:87-89.

3. Bendich, A., Machun, J. L., and Scandurra, O. 1986. The antioxidant role of vitamin C. Adv. Free Radical Biol. Med. 2:419-444.

4. Bestwick, C. S., Brown, I. R., Bennett, M. H. R., and Mansfield, J. 1997. Localization of hydrogen peroxide accumulation during the hypersensitive reaction of lettuce cells to Pseudomonas syringae pv. phaseolicola. Plant Cell 9:209-221.

5. Briggs, R. T., Drath, D. B., Karnovsky, M. L., and Karnovsky, M. J. 1975. Localization of NADH oxidase on the surface of human polymorphonuclear leukocytes by a new cytochemical method. J. Cell Biol. 67:566-586.

6. Daub, M. E., and Ehrenshaft, M. 2000. The photoactivated Cercospra toxin cercosporin: contributions to plant disease and fundamental biology. Annu. Rev. Phytopathol. 38:461-490.

7. Deighton, N., Muckenschnabel, I., Goodman, B. A., and Williamson, B. 1999. Lipids peroxidation and the oxidative burst associated with infection of Capsicum annuum by Botrytis cinerea. Plant J. 20:485-492.

8. Egan, M. J., Wang, Z. Y., Jones, M. A., Smirnoff, N., and Talbot, N. J. 2007. Generation of reactive oxygen species by fungal NADPH oxidases is required for rice blast disease. Proc. Natl. Acad. Sci. USA 104:1177211777.

9. Giesbert, S., Schürg, T., Scheele, S., and Tudzynski, P. 2008. The NADPH oxidase Cpnox1 is required for full pathogenicity of the ergot fungus Claviceps purpurea. Mol. Plant Pathol. 21:808-819.

10. Hayami, C., Otani, H., Nishimura, S., and Kohmoto, K. 1982. Induced resistance in pear leaves by spore germination fluids of nonpathogens to Alternaria alternata Japanese pear pathotype and suppression of the induction by AK-toxin. J. Fac. Agric. Tottori Univ. 17:9-17.

11. Hayashi, N., Tanabe, K., Tsuge, T., Nishimura, S., Kohmoto, K., and Otani, H. 1990. Determination of host-selective toxin production during spore germination of Alternaria alternata by high-performance liquid chromatography. Phytopathology 80:1088-1091.

12. Kodama, M., Wada, H., Otani, H., Kohmoto, K., and Kimura, Y. 1998. 3,5-Di-O-caffeoylquinic acid, an infection-inhibiting factor from Pyrus pyrifolia induced by infection with Alternaria alternata. Phytochemistry 47:371-373.

13. Kumar, J., Hückelhoven, R., Beckhove, U., Nagarajan, S., and Kogel, K.H. 2001. A fungus Bipolaris sorokiniana (Teleomorph: Cochliobolus sativus) and its toxins. Phytopathology 91:127-133.

14. Kwon, B., and Foote, S. C. 1988. Chemistry of singlet oxygen. 50. Hydroperoxide intermediates in the photooxygenation of ascorbic acid. J. Am. Chem. Soc. 110:6582-6583.

15. Lloyd, G., and McCown, B. 1981. Commercially feasible micropropagation of mountain laurel, Kalmia latifolia by use of shoot tip culture. Comb. Proc. Int. Plant Prop. Soc. 30:421-427.

16. Murakami, J., Tosa, Y., Kataoka, T., Tomita, R., Kawasaki, J., Chuma, I., Sesumi, Y., Kusaba, M., Nakayashiki, H., and Mayama, S. 2000. Analysis of host species specificity of Magnaporthe grisea toward wheat using a genetic cross between isolates from wheat and foxtail millet. Phytopathology 90:1060-1067. 
17. Nakashima, T., Ueno, T., Fukami, H., Taga, T., Masuda, H., Osaki, K., Otani, H., Kohmoto, K., and Nishimura, S. 1985. Isolation and structures of AK-toxin I and II, host-specific phytotoxic metabolites produced by Alternaria alternata Japanese pear pathotype. Agric. Biol. Chem. 49:807815 .

18. Nishimura, S., and Kohmoto, K. 1983. Host-specific toxins and chemical structures from Alternaria species. Annu. Rev. Phytopathol. 21:87-116.

19. Nishimura, S., and Kohmoto, K. 1983. Role of toxins in pathogenesis. Pages 137-157 in: Toxins and Plant Pathogenesis. J. M. Daly and B.J. Deverall, eds. Academic Press Australia, Sydney, Australia.

20. O’Donnell, V. B., Tew, D. G., Jones, O. T. G., and England, P. J. 1993. Studies on the inhibitory mechanism of iodonium compounds with special reference to neutrophil NADPH oxidase. Biochem. J. 290:41-49.

21. Otani, H., Kohmoto, K., Kodama, M., and Nishimura, S. 1991. Role of host specific toxins in the pathogenesis of Alternaria alternata. Pages 139-149 in: Molecular Strategies of Pathogens and Host Plants. S. S. Patil, S. Ouchi, D. Mills, and C. Vance, eds. Springer, Berlin, Heidelberg, New York.

22. Otani, H., Nishimura, S., and Kohmoto, K. 1983. Different responses of heatshocked pear leaves to Alternaria alternata Japanese pear pathotype and AK-toxin. J. Fac. Agric. Tottori Univ. 18:1-8.

23. Otani, H., Nishimura, S., Kohmoto, K., Yano, K., and Seno, T. 1975. Nature of specific susceptibility to Alternaria kikuchiana in Nijisseiki cultivar among Japanese pear. (V) Role of host-specific toxin in early step of infection. Ann. Phytopathol. Soc. Jpn. 41:467-476.

24. Rotem, J. 1994. The biotic and physiological components of pathogenesis. Pages 95-118 in: The Genus Alternaria: Biology, Epidemiology, and Pathogenicity. J. Rotem, ed. American Phytopathological Society, St. Paul, MN

25. Segmüller, N., Kokkelink, L., Giesbert, S., Odinius, D., van Kan, J., and Tudzynski, P. 2008. NADPH oxidases are involved in differentiation and pathogenicity in Botrytis cinerea. Mol. Plant-Microbe Interact. 21:808819.

26. Semighini, C., and Harris, S. 2008. Regulation of apical dominance in Aspergillus nidulans hyphae by reactive oxygen species. Genetics 179:1919-1932.

27. Shinogi, T., Suzuki, T., Kurihara, T., Narusaka, Y., and Park, P. 2003. Microscopic detection of reactive oxygen species generation in the compatible and incompatible interactions of Alternaria alternata Japanese pear pathotype and host plants. J. Gen. Plant Pathol. 69:7-16.

28. Tanabe, K., Watanabe, K., Otani, H., and Kohmoto, K. 1991. Chemical characterization and biological activity of the inducer for disease resistance released by Alternaria alternata. J. Fac. Agric. Tottori Univ. 27:1-10

29. Tanaka, S. 1933. Studies on black spot disease of Japanese pear (Pyrus serotina Rehd.). Mem. Coll. Agric. Kyoto Imp. Univ. 28:1-31.

30. Venisse, J. S., Gullner, G., and Brisset, M. N. 2001. Evidence for the involvement of an oxidative stress in the initiation of infection of pear by Erwinia amylovora. Plant Physiol. 125:2164-2172.

31. Walton, J. D. 1996. Host-selective toxins: agents of compatibility. Plant Cell 8:1723-1733

32. Yamamoto, M., Nakatsuka, S., Otani, H., Kohmoto, K., and Nishimura, S 2000. (+)-Catechin acts as an infection-inhibiting factor in strawberry leaf. Phytopathology 90:595-600. 